\title{
Meaning-in-use: Zum Verhältnis von Normativität und Normalität in der Normenforschung
}

Sassan Gholiagha, Hannes Hansen-Magnusson, Maren Hofius

\section{Zur Frage des Verhältnisses von Normalität und Normativität}

Die Frage nach dem Verhältnis zwischen Normalität und Normativität berührt ein Kernproblem der Untersuchung von Ordnung und Wandel in den Internationalen Beziehungen (IB), welche zu stellen konstruktivistische Ansätze möglich gemacht haben (Kratochwil 1989, Onuf 1989). Der analytische Fokus wurde weg von materiellen Interessen einer Maximierung von Sicherheit und Wohlstand zugunsten einer sozialen Ontologie verschoben. Forschende der IB stellten daraufhin zunehmend die Frage, wie und von wem internationale Politik gestaltet wird. Dies bedeutet, dass nicht allein Staaten, schon gar nicht als monolithisches Konzept, für internationale Politik maßgeblich sind, sondern Nichtregierungsorganisationen ebenso zu berücksichtigen sind wie die Rolle, die Individuen innerhalb von Organisationen bekleiden. Makrophänomene wie Staat, globale Ordnung, aber auch Normen erschließen sich nur durch eine Perspektive auf Mikrophänomene (Coulter 2001, Autessere 2014, Bueger 2014, Hofius 2016, Solomon/Steele 2017, Hansen-Magnusson 2020).

Die Parameter der neuen Ontologie sind allerdings bei weitem nicht eindeutig abgesteckt. Im Bereich der Normenforschung, die seit den frühen 1990er Jahren auf das Forschungstableau rückte, zeigt sich konstruktivistische Forschung uneins hinsichtlich der Herangehensweise an Fragen nach Ordnung und Wandel. Diese Debatte soll an dieser Stelle nicht im Detail wiederholt werden, da sie ausführlich von den HerausgeberInnen dieses Sammelbandes und auch von Bastian Loges (dieser Band) skizziert wurde. Wir schließen uns insbesondere der Kritik (Hopf 1998, Reus-Smit 2003, Fierke 2010) einer inkonsequenten Vermischung von sozialer Ontologie (Berger/Luckmann 1991 (1966)) und einer positivistischen Epistemologie an, die sich beispielsweise auf die Suche nach universellen Eigenschaften und Rahmenbedingungen für Akteurshandeln erschöpfte (Wendt 
1992, 1998, Kritisch hierzu: Kratochwil/Ruggie 1986). ${ }^{1}$ Derlei Inkonsistenzen wurden schon früh als Verdinglichung von Normen (Wiener 2003) kritisiert. Derlei Ansätze sind nicht geeignet, das Verhältnis von Normalität und Normativität zu beleuchten, denn um Kriterien zur Messung des Grades der Einhaltung von Normen aufstellen zu können, muss die Frage bereits klar umgrenzte Vorstellungen von Normalität enthalten. Auf diese Weise werden aber im ,Forschungslabor ${ }^{\varsigma}$ Bedingungen für die Wirklichkeit formuliert, für die die folgende Untersuchung Grade der Annäherung verifiziert. $^{2}$

Fast immer wird hierbei übersehen, dass involvierte AkteurInnen - egal ob als Individuum oder Kollektiv - durch Interaktion bedeutungstragende Praktiken miteinander in Bezug bringen und es zunächst im Wesentlichen dieser Prozess ist, der eine Norm entstehen lässt. Ein geteiltes Verständnis entwickelt sich durch die Objektivierung gemeinsamer Erfahrung im intersubjektiven Austausch. Interaktionspraktiken und Sprache tragen also maßgeblich dazu bei, dass Verträge und Konventionen als Objektivierung des Gemeinsamen entstehen können. Nicht umsonst wird in Bezug auf Rechtsnormen in der Wiener Vertragsrechtskonvention in Artikel 32 die Bedeutung der travaux préparatoires ${ }^{3}$ für eine spätere Auslegung von Vertragstexten betont (WVRK 1969). ${ }^{4}$ Diese Art von relationaler Kontextgebundenheit, die das Normalisierende von Normen für die Forschung zugänglich macht, wird übersehen, wenn der Blick lediglich auf Einhaltung und eine möglichst genaue Umsetzung gerichtet ist (Alkoby 2008).

An diese Kritik anschließend gehen wir über die konventionelle Definition von Normen hinaus. Katzenstein definiert Normen als »collective expectations for the proper behaviour of actors with a given identity " Katzenstein 1996: 5). Sie entstehen erstens spontan als soziale Praxis, oder wer-

1 Die Kritik identifiziert einerseits einen mainstream bzw. modern oder auch structural constructivism, der zwar vorgibt, sich im Großen und Ganzen am soziologischen Institutionalismus zu orientieren, aber in seiner Methodologie dem Positivismus behavioristischer Ansätze verhaftet bleibt. Im Gegensatz hierzu wird die mit dem linguistic turn verbundene Methodologie eines critical bzw. consistent constructivism betont, welche die zentralen Forderungen der Dritten Debatte umzusetzen versucht.

2 Es gibt jedoch einige Ansätze, die dem entgegenwirken. Diese zeichnen sich durch ein reflexives Forschungsverständnis aus, beispielsweise in Form von offenen Interviews, ethnographischen Beobachtungen oder Methoden des offenen Codierens.

3 Sie geben Auskunft darüber, wie der Prozess der Entwicklung verlaufen ist und bieten maßgebliche Hinweise für nachfolgende Interpretationen.

4 Nichtsdestotrotz findet sich in der völkerrechtlichen Literatur bis auf wenige Ausnahmen (Brunnée/Toope 2010) ein rechtspositivistisches Normenverständnis. 
den zweitens bewusst als politische Strategien beworben, oder drittens zur Lösung von Konflikten ausgehandelt. Denkbar ist auch eine Kombination dieser drei Möglichkeiten (Katzenstein 1996: 21). Gerade weil Normen zugleich sozial konstruiert als auch soziale Wirklichkeit konstruierend sind, müssen wir davon ausgehen, dass sie in ihrer Bedeutung inhärent umstritten sind (Wiener 2004: 200). So steht im Mittelpunkt dieses Beitrags der Umgang mit Bedeutung, bezeichnet als meaning-in-use (vgl. Weldes/Saco 1996: 373). ${ }^{5}$ Mit meaning-in-use wird sowohl der Umgang mit bestehender Bedeutung in Praktiken bezeichnet als auch Praktiken, die neue Bedeutungen kreieren. Meaning-in-use fordert somit das tradierte Verständnis von Stabilität in den IB heraus ${ }^{6}$ und untersucht Interaktionsprozesse vor dem Hintergrund der Annahme, dass gesellschaftliche Konstellationen wandelbar sind bzw. sich permanent einer Wandlung unterziehen. Dass solche Prozesse von Wandel nicht ohne Auseinandersetzung stattfinden, liegt auf der Hand. So hat Dirk von Gehlen im Februar 2012 in einem Artikel in der Süddeutschen Zeitung am Beispiel der Urheberrechtsdebatte deutlich gemacht, dass für Vertreterinnen und Vertretern beider Seiten in einer Debatte das als Norm erscheint, was für sie in ihrem Kontext normal ist, d.h. jenes Normenverständnis, mit dem sie aufgewachsen sind. Was also ,normal' erscheint, ist immer kontextgebunden. Zu Recht spricht von Gehlen hier bereits im Titel seines Kommentars von der »normativen Kraft des Faktischen« (von Gehlen 2012).

Der hier vorgestellte Ansatz betont exakt jenes Spannungsverhältnis zwischen Normalität und Normativität, indem er das Normative und die andauernde Suche nach dem Normalen in den Blick nimmt. Normen entstehen durch Interaktion und unterliegen einem letztlich niemals erfolgreichen Versuch der Verdinglichung und Essentialisierung, da beteiligte Akteure stets kulturell konnotierte Bedeutungselemente zu Begegnungen mitbringen (Klabbers 2006). Dies soll im Folgenden mit Bezug auf das Folterverbot demonstriert werden: Rechtsnormen sind praxisabhängig und

5 Diese inhärente Umstrittenheit basiert auf der doppelten Qualität von Normen als konstituierend und konstitutiert zugleich. Normen sind, was Akteure aus ihnen machen (vgl. Wiener/Puetter 2009). Wichtig für dieses Verständnis ist auch die Überlegung, dass die Qualität von Normen von der ständigen Anwendung, dem re-enactment, abhängt (Turner 1982; Butler 1997). Der Begriff der Umstrittenheit ist in den letzten Jahren vermehrt aufgegriffen (Welsh 2013; Lantis/Wunderlich 2018; Sandholtz 2019; Deitelhoff/Zimmermann 2020; Hunt/Orchard 2020) und theoretisch weiterentwickelt worden (Wiener 2014; 2018).

6 Stabilität wird vor allem hinsichtlich des strukturellen Kontexts angenommen, beispielsweise Anarchie als Dauerzustand oder aber ein a priori-Rationalismus, der das Streben nach Sicherheit oder Wohlstand als handlungsleitend beschreibt. 
sozio-kulturell konnotiert. Sie unterliegen trotz ihrer Verschriftlichung einem permanenten Wandel. Insofern bezeichnet der Begriff normal etwas temporär Gesetztes, das eine in einem bestimmten Kontext geltende Erwartungshaltung bezüglich des Verhaltens von AkteurInnen produziert, während normativ sich auf einen damit verbundenen, nicht unbedingt spannungsfreien Prozess bezieht, in dem es um (unterschiedliche) Sollensoder Wunschvorstellungen der beteiligten AkteurInnen geht. Normativ beschreibt also ein Desiderat, an das sich gehalten werden soll, aber nicht zwingend gehalten werden muss.

Ziel dieses Beitrags ist es, den erkenntnistheoretischen Mehrwert des meaning-in-use Konzepts für die Normenforschung zu unterstreichen. Im folgenden Abschnitt wird daher zunächst meaning-in-use als zentrales Konzept vorgestellt. Im Anschluss daran werden zwei illustrierende Fallstudien zur Folterverbotsnorm skizziert: zum einen der Umgang mit Gefangenen in Guantanamo Bay, zum anderen die Rechtspraxis des Europäischen Gerichtshofs für Menschenrechte (EGMR). An diesen Fällen wird der durch meaning-in-use produzierte Erkenntniswert deutlich gemacht, nämlich der analytische Zugang zu sich ändernden Bedeutungszuschreibungen durch Praktiken bei prima facie eindeutig kodifizierten (Rechts-) Normen. Gerade im Bereich des Rechts, in dem Normen durch Verschriftlichung vermeintlich fixiert sind, sind Veränderungsprozesse andernfalls nicht plausibel erklärbar.

\section{Meaning-in-use - eine Skizze}

In der sogenannten Dritten Debatte (Wæver 1996) entwickelte sich eine Methodologie, die eine bewusste Reflexion über den Forschungsgegenstand forderte und sich zudem mehr oder weniger explizit an die Kritische Theorie anlehnte. Früh schon waren Friedrich Kratochwil und John Ruggie überzeugt, dass für die IB und Global Governance eine neue Forschungshaltung gefunden werden müsse: »the only viable option [consists of] opening up the positivist epistemology to more interpretive strains, more closely attuned to the reality of regimes (Kratochwil/Ruggie 1986: 766). Ziele eines solchen Ansatzes, der maßgeblich von sprachphilosophischen und hermeneutischen Ansätzen geprägt sein würde (Kratochwil/ Ruggie 1986: 763, 765, Fn.45, 771, Fn.65), sind Erkenntnisse über die "constitution and functioning of regulatory international institutions. They can be used as well to generate insights into the very orders in which institutions are embedded « (Neufeld 1993b: 57). Neufelds Unterscheidung eröffnet die Möglichkeit praxeologischer Untersuchungen sowohl hin- 
sichtlich organisationsinterner Vorgänge als auch kontextbezogener Entwicklungen über einen längeren Zeitraum. Grundlage eines solchen Forschungsansatzes ist die detaillierte Auseinandersetzung mit Sprache und bedeutungstragenden Praktiken, durch welche ein andauernder Prozess von Ordnungsgestaltung angeschoben wird. ${ }^{7}$

Im Kern dieses Ansatzes, der insbesondere durch sogenannte Kritische KonstruktivistInnen für die Forschungspraxis weiterentwickelt wurde, steht ein reflexives Verständnis von Diskurs, wonach in der Forschungspraxis sowohl bedeutungstragende als auch bedeutungsgenerierende Aspekte berücksichtigt werden müssen. Diskurse sind bedeutungstragend, wenn die "symbolic resources out of which the meaningful world is created " gemeint sind (Weldes/Saco 1996: 374, Hervorh. dort). Diskurse sind aber nicht nur bedeutungstragend, sondern zugleich bedeutungsgenerierend. Die Autorinnen schreiben: "Discursive practices, in turn, are the activities through which people create their world and populate it with objects, with subjects, with 'histories and dilemmas', and with dangers" (Weldes/Saco 1996: 374). Die Gleichzeitigkeit von Gegebenem und Entstehendem wird unter dem Konzept des meaning-in-use zusammengefasst (Weldes 1998). Bedeutungsgenerierende Prozesse laufen nicht automatisch ab, sondern sind Teil menschlicher Interaktion. Interaktion ist durch die symbolischen Ressourcen, die zur Verfügung stehen, kontextuell eingebettet (Fierke 2001). Gleichzeitig trägt Interaktion auch zur Neugestaltung des Kontextes bei, indem die Welt mit den schon genannten »objects», »subjects«, »histories and dilemmas" und »dangers « (Weldes/Saco 1996: 374) angereichert wird.

Die Bedeutungszuschreibung umfasst eine Machtkonstellation, die sich in Kategorisierungen ausdrückt. Kategorisierungen tragen zu einer Einteilung und Unterscheidung von Elementen der sozialen Wirklichkeit bei. Sie kreieren Komponenten der Wirklichkeit, setzen sie miteinander in Bezug und hierarchisieren zugleich (Laffey/Weldes 1997: 218-220). Bedeutung entsteht damit durch ein Arrangement von Diskurselementen. Dieses Arrangement unterliegt einer grammatikähnlichen Regelhaftigkeit, die die Forschung rekonstruierend aufdecken kann, ohne dieser jedoch eine Kausalwirkung zuzuschreiben, wie es die positivistische Epistemologie des (Neo-)Liberalismus täte (als Beispiel hierfür vgl. Joerges 2005). So kann man feststellen:

7 Ausführlicher hierzu Hansen-Magnusson (2020: Kap. 1). 
»It makes no sense to say that one term or element of a discourse 'causes' the meaning of another. Instead, it is the relationship between them, as shaped by the rules of a discourse (or grammar) that constitutes their meanings« (Weldes/Saco 1996: 373).

All dies wirft die Frage nach dem Verhältnis von Normen und Diskurs auf (vgl. auch Graf/Glaab/Engelkamp dieser Band). Aus unserer Perspektive entstehen Normen und ihre Bedeutungen im gesellschaftlichen Prozess. Dies gilt gleichermaßen für den Aspekt der inhärenten Umstrittenheit von Normen, wobei der Diskurs gleichsam den Raum der bedeutungstragenden Praktiken (also des Sprechens sowie des nonverbalen Tuns) umfasst (vergl. Holzscheiter 2014; 2017).

Gleichzeitig muss betont werden, dass Prozesse des Definierens und Kategorisierens nicht allein maßgeblich sind, sondern immer auch unmittelbar mit ihrer praktischen Umsetzung in Bezug stehen (Milliken 1999: 241). Erst dadurch ist es möglich, die Bedeutungsgenerierung zu erfassen. Michel Foucault (1977) hat aufgezeigt, wie die Norm ihre Normativität gerade erst durch die normalisierenden, wiederkehrenden Praktiken innerhalb einer Gesellschaft entfaltet. Sie wird deshalb gleichwohl als normal wahrgenommen. Am Beispiel des Gefängnisses erläutert er in »Überwachen und Strafen,$^{8}$ wie die alltägliche Praxis des Strafens durch Differenzierung und Hierarchisierung ein integriertes System entwickelt, das vom Individuum selbst übernommen und verstetigt wird und sich anschließend in Einrichtungen wie Schulen und Krankenhäusern wiederfindet. ${ }^{9}$

Meaning-in-use zielt deshalb auf die Emergenz von Diskursen und damit verbundene menschliche Praxis ab (für eine kritische Diskussion des Ansatzes vgl. Schillinger/Niemann dieser Band und Niemann/Schillinger 2017). Der Ansatz eröffnet neue Fragemöglichkeiten: wie beispielsweise normalisieren sich Kategorien und damit verbundene Prozesse? Wie werden sie hergestellt und wiederverwendet (Laffey/Weldes 1997: 213)? Zu-

8 Es wird im Folgenden auf die englische Ausgabe von 1977 verwiesen.

9 Es muss angemerkt werden, dass Foucault zwar die subtilen Mechanismen individueller Praktiken anerkennt, aber die Frage, wie Wandel überhaupt ermöglicht wird sowie welchen Effekt dieser hat, nicht betrachtet. In ihrer Kritik poststrukturalistischer Forschung geht Fierke (2001) auf eben diesen Punkt ein: »They [Poststrukturalisten] do not sufficiently account for how critical action becomes possible against a disciplining power that primarily reproduces state power and marginalizes alternatives « (Fierke 2001: 121). In Anlehnung an Fierke und im Gegensatz zu Foucault beziehen wir jedoch insbesondere die Veränderungen des Moments durch den Blick auf die Verwendung von Sprache und sich wiederholende Praktiken mit ein. 
gleich ist es aber auch notwendig zu betonen, dass Untersuchungen kontextgebunden bleiben. Die Forschung hat es mit einem andauernden Konstruktionsprozess zu tun, der keinen Abschluss findet (Neufeld 1993a, Doty 1997, Cox 2008). Ergebnisse sind als Zwischenergebnisse eines Forschungsprozesses zu verstehen, der von einer inhärenten Offenheit geprägt ist, wie es auch für den eigenen Untersuchungsgegenstand gilt: Bedeutung ist nie endgültig stabil, sondern bleibt letztendlich offen für Veränderung.

Aus diesem Grund kann auch nicht davon ausgegangen werden, dass eine Norm per se mit Bedeutung aufgeladen ist, die es zu entdecken gilt. Interaktionsprozesse sind grundsätzlich von Indexikalität (Garfinkel 1967) geprägt, was die ex ante Annahme eines geteilten Verständnisses von Normalität schwierig macht (Wiener 2009). Selbst wenn Normen durch Verträge und Konventionen vermeintlich fest-geschrieben sind, bedarf es eines andauernden Interpretationsvorgangs, um zu einer vorübergehenden Klärung zu gelangen (Klabbers 2006, Howse/Teitel 2010). Die AkteurInnen treten dem Text nicht unvoreingenommen gegenüber, sondern sind dabei maßgeblich von ihrem zuvor erworbenen Fundus an Standpunkten geprägt, der als background knowledge, normative baggage oder Horizont (Neufeld 1993b, Adler 2005, Pouliot 2008, Wiener 2008, 2010, Hansen-Magnusson 2020) bezeichnet werden kann. Dieser Vorgang der Textinterpretation, der nun erfolgt, ist ein aktiver sprachlicher Prozess, der gewissermaßen das in-use-Moment betont. VölkerrechtlerInnen, die sich mit interactive international law beschäftigen, unterstreichen die Wichtigkeit von derlei Interaktionsprozessen, da sie maßgeblich zu einer gemeinsamen Verständigung beitragen (Alkoby 2008, Brunnée/Toope 2010). ${ }^{10}$ Der Text einer Konvention oder eines Vertrages, der der Interaktion folgt, repräsentiert die mediale Externalisierung gemeinsamer Verständigung. Anders als es der positivistisch orientierte Mainstream der Global Governance-Forschung jedoch betonen würde, ist dies weder der Endpunkt einer Normentwicklung noch der Beweis für eine endgültige Bedeutungs-fest-schreibung [sic]. Vielmehr ist der Text nun Teil jener symbolischen Ressourcen, mit denen Wirklichkeit weiterführend hervorgebracht werden kann. Das Arrangement der Diskurselemente kann Änderungen unterworfen sein, wodurch sich wiederum Bedeutungsänderungen ergeben. Praktiken unterscheiden sich, möglicherweise kommt es zu neuen Versuchen einer Festschreibung, so dass der Prozess der Bedeutungsgenerierung nicht als abgeschlossen betrachtet werden kann.

10 Der Ansatz ist auch auf den Bereich der Internationalen Politischen Ökonomie übertragbar, z.B. bei Park/Vetterlein (2010). 
Im Folgenden wird nun anhand zweier illustrativer Fallbeispiele der oben skizzierte Ansatz des meaning-in-use weiter ausgeführt. Dabei liegt der Fokus im ersten Fallbeispiel zum Umgang mit Gefangenen in Guantanamo Bay auf den Praktiken von Akteuren (monologisch). Der Fokus des zweiten Fallbeispiels zur Diskussion um das Folterverbot in der Europäischen Menschenrechtskonvention liegt auf den Aushandlung von Bedeutung (dialogisch). Beides zusammen genommen erlaubt so eine umfassendere Analyse von meaning-in-use im Kontext der Debatte um Folter und Folterverbot.

\section{Zur Normalisierung der Folterpraxis}

Insbesondere in Bezug auf das häufig perzipierte Spannungsverhältnis zwischen dem verschriftlichten Recht und sozialer Praktiken verdeutlicht ein Rückgriff auf Foucaults Konzept der Disziplinarmacht die Verflechtung von Normalität und der normativen Qualität von Normen. Diese wird sichtbar, da er den analytischen Fokus auf normalisierende Praktiken lenkt, welche einen kontinuierlichen Prozess der Normalisierung anstelle einer ontologisch gegebenen Normalität abbilden. Normalisierende Praktiken als meaning-in-use sind demnach das verbindende Element zwischen Normalität und Normativität. So zeigt Foucault auf, wie Individuen, die durch alltägliche Praxis eine Disziplin verinnerlicht haben, als Richter des Normalen agieren, auf deren Schultern gleichwohl »the universal reign of the normative is based « (Foucault 1977: 304, Hervorh. dort). Das Normative entspricht hier stets dem kontingenten Resultat eines Aushandlungsprozesses, welcher sich durch das Definieren und Kategorisieren von Objekten auszeichnet. Durch solche Praktiken kann sich dann ein sogenanntes Gegenrecht zum ordentlichen Recht etablieren (Foucault 1977: 222, 224), welches die Bedeutung des Rechts einem Wandel unterzieht. ${ }^{11}$

Der folgende Abschnitt soll am Beispiel des US-Gefangenenlagers Guantanamo Bay in Kuba verdeutlichen, wie die dortigen Menschenrechtsverletzungen und Verstöße gegen das Humanitäre Völkerrecht unter der

11 Wie genau Foucault die Unterscheidung zwischen Recht und entstehendem Gegenrecht unternimmt, bleibt unklar; weiterhin ist nicht erwähnt, durch welche Prozesse das ordentliche Recht entsteht. Die unscharfe Trennung zwischen den beiden Begriffen mag jedoch beabsichtigt sein, zieht man in Betracht, dass das Gegenrecht sich genau in all jenen grauen Zwischenräumen entfaltet, welche von der binären Gut-Böse-Einteilung des Rechts nicht erfasst werden (vgl. Foucault 1977: 180). 
Bush-Administration (als auch in abgemilderter Form unter der ObamaAdministration) durch die Perpetuierung eines normativ wie institutionell dichten Netzwerkes (Johns 2005: 618) von Regulationen und eigens errichteten Tribunalen, Kommissionen und Gremien möglich waren. Die von der Bush-Administration minutiös niedergeschriebenen und eingeführten Praktiken wie die Verhörmethoden (coercive interrogation) erlaubten, international geltendes (Humanitäres) Völkerrecht durch ihre spezifische Auslegung in Guantanamo vor dem Hintergrund eines Ausnabmezustandes zu unterlaufen und eine über ihren Kontext hinaus geltende Normumdeutung des Folterverbots vorzunehmen. ${ }^{12}$ So gelang es, vormals rechtswidrige Praktiken innerhalb des internationalen Rechts als das geltende, normativ notwendige Recht zu etablieren. ${ }^{13}$

\subsection{Regulierung obne Rechte}

»The plight of the Guantánamo detainees is less an outcome of law's suspension or evisceration than of elaborate regulatory efforts by a range of legal authorities. The detention camps are above all works of legal representation and classification. They are spaces where law and

12 Vgl. hierzu auch die Wirkung, die die Praktiken der Bush Regierung für autoritär geführte Regime seit 2002 hatten. Unter dem Vorwand des War on Terror fanden Letztere eine Legitimierungsquelle für ihre bis dato international geächteten Verhör- bzw. Foltermethoden.

13 Es mag durchaus berechtigt sein, die Anwendung Foucaults in Guantanamo zu hinterfragen, gleichen doch dort die Praktiken einer absolut aufgezwungenen Gewalt anstelle einer sich selbst-erzwingenden Disziplin, die in aller Stille operiert. Das Gegenteil ist der Fall in Guantanamo, denn »the obsession with torture does not breed in darkness, in the secret shadows of the state; it flowers in the threshold between the legal and the extra-legal«, so Derek Gregory (2006: 420). Dennoch wäre eine frühzeitige Verwerfung Foucault'scher Überlegungen hier nicht angebracht. Gerade die Herausarbeitung von Praktiken wie die zusätzliche Regulierung oder Normierung nach Foucault können darüber Aufschluss geben, wie Guantanamo Bay in einem liberal-demokratischen Staat wie den USA überhaupt möglich werden und durch die institutionelle Verstetigung von Praktiken eine punktuelle Normalisierung erfahren konnte. $\mathrm{Zu}$ einer weiteren Anwendung Foucault'scher Konzepte im Falle Guantanamos vgl. auch Neal (2006), der mithilfe der Methode der Archäologie die Verstetigung von Binärstrukturen wie Normalität vs. Ausnahme offenlegt und kritisiert, welche sich in der wissenschaftlichen Debatte u.a. in Agambens viel zitiertem Werk »Ausnahmezustand « (2005) niederschlagen. 
liberal proceduralism speak and operate in excess « (Johns 2005: 614, Hervorh. dort).

Als der US-Marinestützpunkt auf Guantanamo Bay in den 1990er Jahren noch als Internierungslager für haitianische und kubanische Flüchtlinge genutzt wurde, nannte Gerald Neuman es 1996 eine "anomalous zone" (Ahmad 2009: 1709), da die damalige US-Regierung den Asylanten schon in dieser Zeit jedweden Rekurs auf verfassungsrechtlichen Schutz verwehrte. Seit Januar 2002, als die Bush-Administration im Zuge ihrer Militärkampagne in Afghanistan hunderte Verdächtige als vermeintliche Al Qaeda-Mitglieder nach Guantanamo Bay einfliegen und internieren ließ, wurde das Argument, Guantanamo gleiche einem rechtsfreien Raum, insbesondere von JuristInnen immer wieder verwendet.

Diese Einschätzungen basieren darauf, dass die Bush-Administration 2001 den Gefangenen den Kriegsgefangenstatus verwehrte und so die Gültigkeit der dritten Genfer Konvention unterband. Stattdessen wurden sie als enemy oder unlawful combatants eingestuft - ein Terminus, der laut Ahmad eine „rechtliche Erfindung“ (2009: 1705) darstellt bzw. ein völkerrechtlich nicht anerkannter Begriff ist (Venzke 2009). Seit März 2009 wurde dieser Begriff jedoch unter der Obama-Administration wieder zurückgenommen. Dies gilt ebenso für die Verwerfung aller Folter-Memos. Das erste Mal 1942 im Fall Ex Parte Quirin durch den US Supreme Court verwendet, fand der Begriff unlawful combatant dann wieder vermehrt durch Rechtsakte wie den im September 2001 gefassten Beschluss des Kongresses Authorization for Use of Military Force Against Terrorists (AUMF) sowie die im November 2001 folgende Presidential Military Order: Detention, Treatment, and Trial of Certain Non-Citizens in the War against Terrorism Legitimierung. Während im Jahre 2004 der Supreme Court in Rasul v. Bush schon einmal den Gefangenen bestätigte, sie hätten ein Recht auf eine gerichtliche Rechtsprüfung ihrer Inhaftierung (babeas corpus writ), verstrichen weitere vier Jahre, inklusive des vom Kongress verabschiedeten Military Commission Act 2006, ${ }^{14}$ bis der Supreme Court 2008 in Boumediene v. Bush die Praxis als verfassungswidrig erklärte.

Innerhalb dieser Zeit war Guantanamo weder eine rechtsfreie Zone noch waren die Inhaftierten, wie Agamben es sagt, »legally unnameable and unclassifiable beings« (in Gregory 2006: 407). Ganz im Gegenteil - darauf verweist Fleur Johns - wurden (und werden weiterhin) die Gefange-

14 Hier stellte sich der Kongress auf die Seite der Bush-Administration und verweigerte jedem als enemy combatant erklärten Inhaftierten das Recht auf richterliche Haftprüfung vor zivilen US-Gerichten. 
nen einer Klassifizierung unterzogen, nicht nur durch die Einstufung als enemy combatant (Johns 2005: 617). Wie Foucault in Überwachen und Strafen die Praktik der normierenden Sanktion beschreibt, so zeigt Gregory (2006) auf, wie der Fortschritt eines Inhaftierten in Guantanamo Bay nach dem Grad der Kooperation gemessen wird: je nachdem, wie kooperativ sich der Gefangene zeigt, steigt er in der Hierarchie auf oder ab und erhält mehr bzw. weniger Privilegien. Diese Einteilung schlägt sich wiederum in dem Ort der Internierung nieder, d.h. die bestehenden Zell-Blöcke Camp Deltas etwa, in denen die enemy combatants untergebracht sind, verfügen über unterschiedliche Stufen von Einschränkungen und Vorrechten (Gregory 2006: 414-415).

Des Weiteren geht es in Guantanamo grundsätzlich darum, das Bild eines von allen Beteiligten möglichst entfernten, auf Fakten basierten Prozesses aufrecht zu erhalten, während dieser von einer inhärent normativen Struktur, also mit Referenzen zur Verteidigung von liberal-demokratischen Rechten, umgeben ist (Johns 2005). Zweifelsohne, so belegen es die Memoranden - die sogenannten Torture Memos - von den beratenden Anwälten des Weißen Hauses oder des US-Verteidigungs- oder Justizministeriums, haben hochrangige Individuen wie Verteidigungsminister Rumsfeld geltendes (Humanitäres) Völkerrecht durch die Zulassung von bis dato in den USA untersagten Foltermethoden ${ }^{15}$ mit Verweis auf deren Notwendigkeit zur Verteidigung dieser Rechte stark gedehnt (Bybee 2002). Unter der Bedingung der Notwendigkeit hatte Assistant Attorney General Jay Bybee argumentiert, den expliziten Einsatz von Verhörmethoden zu erlauben und Folter nur im engsten Sinne auszuschließen. So definierte er Folter nur als der »most severe pain «, der mit »death, organ failure, or serious impairment of body functions" einhergehe und/oder mentalem Leiden über Monate oder Jahre gleichkomme (Bybee 2002: 6).

Es ist signifikant zu sehen, wie allgegenwärtig und genau ausgearbeitet die angewandten Techniken und Mechanismen der inhumanen Behandlung und Folter der detainees waren und so $\mathrm{zu}$ einem elaborierten "governance regime" (Ahmed 2009: 1683) entwickelt wurden, welches von allen drei Gewalten im Prozess fortgesetzt wurde und zur Normalisierung dieser neuen Rechtsauslegung führte. Zunächst erfolgte die Einstufung eines Gefangenen als enemy combatant bei der Gefangennahme nach einem

15 Vgl. z.B. das Memorandum for Commander, Joint Task Force 170, das verschärfte Verhörmethoden vorsah, die durch das berüchtigte Bybee Memo (Standards for Conduct for Interrogation under 18 U.S.C. 2340-2340A) rechtlich auf den Weg gebracht wurden. 
»elaborate, multi-stage screening and evaluation process" (Johns 2005: 617). Zudem wurden Institutionen wie die Military Commissions einberufen oder das Administrative Review Board sowie das Combatant Status Review Tribunal eingerichtet. Bei letzterem wurden Standardskripte für die Anhörung eines Gefangenen für dessen Statusbestimmung vorgeschlagen, um das "Management innerhalb des Verteidigungsministeriums zu verbessern « (Department of Defence 2004: 4). Das neu eingerichtete Office of Detainee Affairs im Pentagon, das beauftragt wurde, die Behandlung der Inhaftierten zu bewerten, ist nach den Worten des Principal Deputy des Under Secretary of Defense for Policy, C. Ryan Henry, vor allem als Versuch $\mathrm{zu}$ verstehen, eine "coherent and seamless policy on detainees « zu haben (zitiert in Porth 2004: para 7).

Johns spricht gar von einem in der Exekutive perzipierten »institutional demand for standardization" (Johns 2005: 630), wenn sie den Pressebericht des Secretary of the Navy, Gordon England, zum alljährlichen Begutachtungsverfahren (administrative review process) der Inhaftierten in 2004 wiederholt:

»[W]e do have some guidelines; ... the boards do have some guidelines', he assured the audience, '[e]very board doesn't have a different standard'. He continued: '[I]t will be a judgment based on facts, data available ... the best decision a reasonable person can make in this situation. [...]. '[I]t's what is the situation today and going forward in terms of a threat to America. And that is what we will decide, and that's what the decision will be based on.' «(Johns 2005: 630)

Weitgehend losgelöst von der individuellen Entscheidung erscheint so das Vorgehen bzw. die Behandlung der Gefangenen als rational, begründet in der Notwendigkeit, die Sicherheit Amerikas zu wahren. Diese Entscheidungen entsprechen einer Logik, die die Lage eines bewaffneten Konflikts - jenseits der Gültigkeit der III. Genfer Konvention - als puren Fakt essentialisiert, daraufhin einen außergewöhnlichen Notstand ausruft und somit die Ausübung von Autorität, inklusive Folter, legitimiert. Das Ergebnis ist nicht nur eine Normalisierung des Notstandes, dessen administrative Praktiken vor allem auf zuvor genehmigten Memoranden wie die sogenannten Torture-Memos fußen. Es ist gleichzeitig eine Normalisierung des Gegenrechts. Dies bedeutet, dass die spezifische Auslegung der Definition von Folter durch Berater des Präsidenten George W. Bush oder des Verteidigungsministeriums wie Assistant Attorney General Jay Bybee sich in routinierten Praktiken vor Ort niederschlugen. Es folgte somit der Versuch einer Festschreibung dieser Interpretation in Guantanamo Bay im Sinne des meaning-in-use Konzepts. 
Foucaults Beschreibung der Macht der Norm hilft uns zu verstehen, wie die punktuelle soziale Fixierung von bestimmten Praktiken ein kodifiziertes Recht herausfordern und anschließend im Rahmen einer permanenten Ausnahme wie dem nationalen Notstand Recht durch Gegenrecht regulieren kann. Eine Insel, die also zunächst als rechtliches Niemandsland deklariert wird, ist im Grunde keineswegs außerhalb des Rechts. Vielmehr ist sie gefüllt mit gesetzlicher Regelung, die sich allerdings außerhalb der Parameter des regulären Rechts befindet: so wird argumentiert, dass andere Rechte gälten, da es sich bei den Gefangenen nicht um Zivilisten (sic), sondern um unlawful combatants handele. Letztlich hilft uns Foucault das Paradoxon zu verstehen, wie ein place of rightlessness in einem Kontext emergieren kann, der keinesfalls lawless ist. ${ }^{16}$ Das durch soziale Praktiken entstehende und sich (ständig) wandelnde Gegenrecht muss also nicht im Gegensatz zu Recht verstanden werden - sei es das einer nationalen Verfassung oder solches, das als verbindliches Völkerrecht gilt. Es repräsentiert vielmehr die Praxis der politischen Entscheidung über eine oder mehrere Rechtsnormen, kurz: Es steht für eine momentane Auslegung der Rechtsnormen, die durch die Regulierung politisch autorisierter Individuen sichtbar wird. Recht und Gegenrecht arbeiten demnach in Johns Worten win tandem with [one another] "(Johns 2005: 634). Recht ist somit nicht selbst-definierend, sondern eine Bedeutungsstruktur ${ }^{17}$ (vgl. Cover 1983), die sich durch die Deutung von Akteuren qua Praxis konstituiert.

Gegenrecht (re)definiert demnach den Inhalt dieser Bedeutungsstruktur durch die Präzision und Genauigkeit spezifischer Praktiken. Übertragen auf das meaning-in-use Konzept zeigt eine Berufung auf Foucault daher, wie die Bedeutung einer Norm durch die Anwendung minutiöser Praktiken verändert und mit (neuem) Inhalt gefüllt werden kann. So kann es, wie am Beispiel des Umgangs mit sogenannten illegal combatants in Guantanamo Bay zu erkennen ist, dazu kommen, dass sogar innerhalb einer jus cogens-Norm wie dem Folterverbot dieser Charakter in der Praxis durch zusätzliche Regulierung unterlaufen wird und dennoch die Neudefinierung in einem bestimmten Kontext als das Normale angesehen wird. Dies bedeutet jedoch nicht, dass das Folterverbot an sich, d.h. der Normtyp hinterfragt wird. Inhärent umstritten ist die Auslegung, also das meaning-in-use.

16 Vergleiche Gregory (2006: 412).

17 Recht wird von Cover (1983: 12) als "system of meaning rather than an imposition of force " definiert, vgl. insbesondere Ahmad (2009: 1693), der sich auf Covers Nomos and Narrative bezieht. 


\subsection{Meaning-in-use, das Verbot der Folterpraxis und die Rechtsprechung des EGMR}

Die im vorangegangenen Abschnitt beschriebenen Versuche der US-Administration der Normalisierung spezifischer Praktiken finden sich ähnlich gelagert auch in den Verhandlungen bezüglich der Rechtspraxis des Europäischen Gerichtshofs für Menschenrechte (EGMR). Auch hier lässt sich ein seitens der politischen Akteure vorgenommener Versuch identifizieren, mit Rückgriff auf tatsächliche oder wahrgenommene Bedrohungen durch Terrorismus eine anderweitige Bedeutungszuschreibung der Folterverbotsnorm vorzunehmen. Interessant hierbei ist, dass sich solche Prozesse in einigen liberalen Demokratien nachweisen lassen, also gerade dort, wo solche Prozesse nach mainstream-KonstruktivistInnen nicht zu erwarten sind (Jepperson et al. 1996; Risse et al. 1999). Dass aber eine liberal community of states nicht vorausgesetzt werden kann, hat bereits Andrea Liese anhand der Fallbeispiele Großbritanniens, Israels und den USA gezeigt (Liese 2009). ${ }^{18}$

Im Folgenden soll anhand eines spezifischen Falls vor dem Europäischen Menschengerichtshofs (EGMR) nachgezeichnet werden, wie die gleiche Norm des Folterverbots in unterschiedlichen Kontexten unterschiedliche Bedeutungszuschreibungen erfährt. Es lässt sich parallel zu Liese (2009) der Versuch identifizieren, anhand von Stellungnahmen und Gerichtsverhandlungen die Absolutheit des Folterverbots anders zu verstehen und $z u$ interpretieren und damit zugleich ein neues Anwendungsverständnis herzustellen. Auch mit Blick auf die oben genannte detaillierte Auseinandersetzung mit Sprache und bedeutungsvollen Praktiken erscheint der Blick auf die Rechtspraxis des EGMR und die im Folgenden vorgenommene detaillierte Analyse von Argumenten innerhalb der Gerichtsverfahren und Argumentationsmuster des Gerichtes selber als lohnendes Anwendungsbeispiel. Anders ausgedrückt geht es um die Frage, wie das Folterverbot durch staatliche Akteure in ihrem Interaktionsprozess miteinander re-

18 Auch in Deutschland hat es eine solche Debatte bereits im Rahmen der staatlichen Maßnahmen gegen die RAF gegeben. Eine neuere Diskussion entbrannte nach der Androhung von Gewalt in einem Verhör mit dem mittlerweile verurteilten Entführer von Jakob Metzler; vgl. Spiegel Online (2004). In einem anschließendem Verfahren vor dem EGMR, das 2010 entschieden wurde, stellte der Gerichtshof fest, dass die Gewaltandrohung in der Tat ein Verstoß gegen $\mathbb{3}$ der EGMR dargestellt hatte (Gäfgen v. Germany. Verfügbar hier: http:// hudoc.echr.coe.int/eng?i=001-99015O). 
enacted wird. ${ }^{19}$ In den konkreten Fällen geschieht dies durch Abschiebeversuche mit Hinweis auf die nationale Sicherheit und eine geänderte Sicherheitslage durch den globalen Terrorismus. Zugleich zeigt die Analyse, dass das Konzept des meaning-in-use ein besseres Verständnis der Umstrittenheit von Normen herstellen kann, welche formal fest in den völkerrechtlichen, europäischen und nationalen Menschenrechtsregimen als jus cogens-Normen etabliert sind. Darüber hinaus wird durch die Analyse die bereits erwähnte Kontextabhängigkeit von Normen deutlich.

\subsection{Das Folterverbot im Kontext der Europäischen Menschenrechtskonvention (EMRK)}

Zunächst einmal soll kurz die rechtliche Grundlage innerhalb der EMRK (1950) dargestellt werden. In $\$ 3$ EMRK heißt es: »Niemand darf der Folter oder unmenschlicher oder erniedrigender Strafe oder Behandlung unterworfen werden.«Von Relevanz ist hier ebenfalls $\$ 15$ EMRK, der das »Abweichen im Notstandsfall« beschreibt und in dessen Absatz 2 festgelegt wird, dass vom absoluten Folterverbot in keinem Fall abgewichen werden kann. Dies beinhaltet explizit Kriegs- und Notstandssituationen, wie wir sie zumindest medial oder politisch im so genannten War on Terror finden.

Bevor auf den im Detail zu analysierenden Fall Saadi v. Italy eingegangen werden kann, bedarf es einer kurzen Darstellung der Rechtsgeschichte des $₫ 3$ und seiner Auslegung. Die Möglichkeit, dass $\$ 3$ EMRK auch Verpflichtungen jenseits des Geltungsbereiches der Konvention mit sich bringt, verdeutlichte der Gerichtshof in Soering v. United Kingdom ${ }^{20} 1989$. Hier findet sich einer der deutlichsten Kommentare zum Stellenwert von $\$ 3$ in der EMRK:

»This absolute prohibition of torture and of inhuman or degrading treatment or punishment under the terms of the Convention shows

19 Wiener spricht hier von re/enacting of the normative structure of meaning-inuse" (Wiener 2009: 176).

20 Im Fall Soering befasste sich der EGMR erstmals mit der Frage der Auslieferung an Drittstatten, wenn dort der auszuliefernden Person Maßnahmen drohten, die unter $\$ 3$ EMRK fielen. Soering war in den USA des Mordes angeklagt, ihm drohte die Todesstrafe. Unter Berufung auf $₫ 3$ EGMR argumentierte er, dass die lange Wartezeit (bis zu 8 Jahre) vor Vollstreckung der Todesstrafe unmenschliche Behandlung bzw. Strafe darstellte (Janis et al. 2008: 215-216). 
that Article 3 (art 3) enshrines one of the most fundamental values of the democratic society making up the Council of Europe. $\ll^{21}$

Dass diese Verpflichtungen auch dann gelten, wenn Bedrohungen durch den internationalen Terrorismus prima facie mit den Rechten, die unter der EMRK gewährt werden, abgewogen werden müssen, verdeutlichte der EGMR nochmals 1996 in Chahal v. United Kingdom: $:^{22}$

»The Court is well aware of the immense difficulties faced by States in modern times in protecting their communities from terrorist violence. However, even in these circumstances the Convention prohibits in absolute terms torture or inhuman or degrading treatment or punishment, irrespective of the victim's conduct. ... In these circumstances the activities of the individual in question, however undesirable or dangerous, cannot be a material consideration. $\ll^{23}$

Ein weiterer Diskurs über die Bedeutungszuschreibung findet hinsichtlich der Frage nach negativen und positiven Pflichten statt, die sich aus dem absoluten Folterverbot für Staaten ergeben. Eine negative Pflicht aus $₫ 3$, also selbst nicht zu foltern, lässt sich relativ eindeutig aus dem Artikel selbst und aus der allgemeinen Rechtsprechung hierzu ableiten. Die aus $\$ 3$ abzuleitenden Verpflichtungen, aktiv Folter sowie inhumane und degradierende Behandlungen zu unterbinden und zu verhindern, leiten sich aus einer Reihe von Fällen vor dem EGMR ab (Palmer 2006).

\section{Herausforderungen durch die Bedrohung durch Terrorismus - Changed Meaning and Changed Use?}

Während in den Guantanamo- und Abu Ghraib-Fällen die Frage nach der Bedeutung von Folter an sich im Fokus stand, ist die Rechtsprechung des EGMR zu $₫ 3$ vor allem mit der Definition des Gültigkeitsbereichs der Norm befasst (wie Verhörmethoden, Prügelstrafe, Haftbedingungen, Auslieferungen und Bedingungen im Empfängerland, Abwägungsfragen mit anderen Rechten der Konvention). Ein starres Normenverständnis kann diese unterschiedlichen Anwendungsfälle mit jeweils eigenen Auslegun-

21 Soering $v$. United Kingdom [1989] 11 EHRR 439, para 88.

22 Im Fall von Chahal ging es um die Auslieferung mit der Begründung, Chahals Anwesenheit in Großbritannien stelle aufgrund seiner politischen Aktivitäten eine Gefährdung der nationalen Sicherheit dar (Janis et al. 2008: 219-220).

23 Chabal v. United Kingdom [1996] 23 EHRR 413, paras 79-80. 
gen nur bedingt nachvollziehen. Erst durch ein Akteurs- und kontextbasiertes Normenverständnis werden die andauernden Auslegungsdebatten vor dem EGMR empirisch wie theoretisch greifbar.

Im Folgenden soll anhand des bereits erwähnten Falls Saadi v. Italy ${ }^{24}$ aus dem Jahr 2008 gezeigt werden, dass Interaktionsprozesse maßgeblich sind für die Emergenz und Bedeutungszuschreibung von Normen. In den beschrieben Fall wie auch in Saadiv Italy geht es letzten Endes auch um den Versuch von Regierungen, die Sicherheit und das Leben der eigenen Bevölkerung im Kontext der Bedrohung der nationalen Sicherheit höher zu bewerten als die Sicherheit der abzuschiebenden Person. Hier findet also wiederum eine wie bereits oben angesprochene Kategorisierung statt, die jedoch in der konkreten Situation seitens des Gerichts nicht nur hinterfragt, sondern abgelehnt wird. Der Fall lässt sich einerseits im Rahmen von Abschiebefällen und gleichermaßen im Kontext von Bedrohungen der nationalen Sicherheit verstehen. Letzteres ist insbesondere seit den Anschlägen vom 11. September 2001 relevant, schien bis dato doch ein übergreifender Konsens bezüglich des Folterverbotes zu existieren. Jedoch hat Nowak in seiner Analyse des absoluten Folterverbots auf globaler Ebene festgestellt:

»The global threat of terrorism at the beginning of the $21^{\text {st }}$ century and the counter-terrorism strategies of the United States, many European and other governments, seem to challenge this universal consensus." (Nowak 2005: 674)

Diese Herausforderung hat unter anderem dazu geführt, dass Regierungen versucht haben, die Bedeutung des absoluten Verbots von Folter zu re-interpretieren, und zwar mithilfe der Rechtsprechung des EGMR. Dem voran gingen diskursive Interventionen (Fierke/Wiener 1999: 674; Milliken 1999) von Regierungsvertretern. So forderte Charles Clarke, Home-Secretary unter Tony Blair, in einer Rede vor dem Europäischen Parlament im Jahr 2005 eine genaue Überprüfung der in der Konvention verbrieften Rechte. Moeckli argumentiert diesbezüglich:

»The main target of the British government's effort 'to rebalance' the European Convention on Human Rights (ECHR), it turned out, was Article 3, the prohibition of torture and inhuman or degrading treatment.« (Moeckli 2005: 534-535)

24 Eine Einordung des Falles in den Kontext einer möglichen Entwicklung der EKMR hin zu einer kosmopolitischen Rechtsordnung bietet Alec Stone Sweet (2012). 
In Chahal hatte die britische Regierung argumentiert, dass angesichts der terroristischen Bedrohung die nationale Sicherheit mit dem Risiko einer unwürdigen Behandlung eines Nicht-Staatsbürgers bei Auslieferungsverfahren abzuwägen sei. Während in Soering sich zumindest auf den ersten Blick eine Grundlage für eine solche Abwägung finden lässt, zeigt Chahal deutlich die Zielsetzung des Gerichtshofs, dies genauer zu regulieren (Palmer 2006: 447). Die Entscheidung des EGMR zeigt dann auch deutlich, dass die RichterInnen keinen Raum für eine solche balancing exercise sahen. Das Urteil in Soering als Folie nutzend, argumentierte der Gerichtshof:

"It should not be inferred from the Court's remarks concerning the risk of undermining the foundations of extradition ... that there is any room for balancing the risk of ill-treatment against the reasons for expulsion in determining whether a State's responsibility under Article 3 (art 3 ) is engaged. $\aleph^{25}$

Eben jene Ablehnung einer Abwägung wurde erneut in Saadi v. Italy ${ }^{26}$ debattiert. Der Kläger war ein tunesischer Staatsbürger, den Italien nach Tunesien ausliefern wollte. Herr Saadi, seinerzeit wegen terroristischer Akte angeklagt, war wegen krimineller Verschwörungen verurteilt worden. Nach seiner Entlassung aus der Haft wollte Italien ihn deportieren. Zeitgleich wurde der Kläger in Tunesien von einem Militärgericht in seiner Abwesenheit zu 20 Jahren Gefängnis wegen terroristischer Aktivitäten verurteilt. Die tunesische Regierung garantierte Italien nach Anfrage, dass Herr Saadi einen fairen Prozess erhielte und keine unwürdige Behandlung befürchten müsse. ${ }^{27}$

Nachdem die britische Regierung bei Chahal gescheitert war, intervenierte sie basierend auf $\$ 36(2)$ EMRK als third party. Die Argumente der britischen Regierung sind deshalb von besonderem Interesse, als sie direkt die in Soering ${ }^{28}$ und Chabal etablierten Prinzipien anzweifelte. In den Einreichungen der britischen Regierung lassen sich vier Argumente identifizieren (Moeckli 2008: 541-544): Das erste Argument bezog sich auf das Recht auf Leben ( $\$ 2$ der Konvention). Die Regierung argumentierte hier,

25 Chahal v. United Kingdom [1996] 23 EHRR 413, para 81; (unsere Hervorhebung).

26 Saadi v. Italy [2008] 24 B.H.R.C. 123.

27 Eine umfassende Darstellung des Falls sowie der Bekundungen der tunesischen Regierung findet sich in Saadi v. Italy [2008] 24 B.H.R.C. 123, paras 9-55.

28 Auch im Fall von El-Masri v. The Former Yugoslav Republic of Macedonia bezog sich der EGMR auf Soering und die Verantwortung des abschiebenden Staates (eine Analyse findet sich bei Nollkaemper (2012)). 
dass bessere Maßnahmen notwendig und die Verpflichtung des Staates, seine Bürgerinnen und Bürger zu schützen, von Bedeutung sein müsse. ${ }^{29}$ Das zweite Argument bezog sich auf die implied positive obligation. In den Worten der britischen Regierung:

"Yet in the field of implied positive obligations the Court had accepted that the applicant's rights must be weighed against the interests of the community as a whole. « ${ }^{30}$

Die weiteren Argumente stellten beide Herausforderungen für den vom Gerichtshof eingeschlagenen Weg bei Chahal dar:

"In the first place, the threat presented by the person to be deported must be a factor to be assessed in relation to the possibility and the nature of the potential ill-treatment. Secondly, national security considerations must influence the standard of proof required from the applicant. In other words, if the respondent State adduced evidence that there was a threat to national security, stronger evidence had to be adduced to prove that the applicant would be at risk of ill-treatment in the receiving country. ${ }^{31}$

Während das $₫ 2$-Argument mit Ausnahme einer abweichenden Meinung von Judge Mijye unter Bezugnahme auf Osman v. United Kingdom ${ }^{32}$ vom EGMR ignoriert wurde, äußerte sich der Gerichtshof deutlicher zu der Frage der Abwägung: "without directly addressing the nature of the obligation, the Grand Chamber refused to draw a distinction« (Moeckli 2005: 542 ).

»[The Court] must therefore reaffirm the principle stated in the Chahal judgment ... it is not possible to weigh the risk of ill treatment against the reasons put forward for the expulsion in order to determine whether the responsibility of a State is engaged under Article 3, even where such treatment is inflicted by another State. «33

Auffällig ist hier die gewählte Formulierung des Gerichts: »[The Court] must reaffirm ..." (unsere Hervorhebung), in der deutlich wird, dass sich der EGMR zu einer erneuten Klarstellung gezwungen sah. Gleichermaßen lehnte der EGMR die Abwägung zwischen individuellen Rechten und na-

29 Saadi v. Italy [2008] 24 B.H.R.C. 123, para 119.

30 Saadi v. Italy [2008] 24 B.H.R.C. 123, para 120.

31 Saadi v. Italy [2008] 24 B.H.R.C. 123, para 122.

32 Osman v. United Kingdom [2000] 29 EHRR 245.

33 Saadiv. Italy [2008] 24 B.H.R.C. 123, para 138. 
tionaler Sicherheit sowie unterschiedlicher Beweispflichten ab. Chabal zitierend, stellte der Gerichtshof fest, dass aufgrund der Absolutheit von dem in $\$ 3$ festgelegten Folterverbots schlichtweg kein Raum für Abwägungen bestand. ${ }^{34}$ Zusammenfassend kann also argumentiert werden, dass auch die jüngeren Versuche liberaler Demokratien ein neues meaning-inuse des Folterverbots im europäischen Rechtsraum zu etablieren, vorerst gescheitert sind. Dieses Scheitern lässt sich zum einen dadurch erklären, dass der EGMR ein gewisses Interesse hat, eine Kontinuität seiner Rechtsprechung aufrecht zu erhalten. ${ }^{35}$ Andererseits wird in den Urteilen des Gerichts auch ein klares Bekenntnis zu unveräußerlichen Menschenrechten deutlich. Nichtsdestotrotz gilt auch hier, was Andrea Liese mit Blick auf die internationale Rechtsentwicklung festgestellt hat, nämlich die Tatsache, dass Akteure sich zwar einerseits an das Folterverbot gebunden fühlen, aber die Bedeutung von Folter an sich und die Reichweite des Verbots dennoch umstritten bleiben (Liese 2009: 45). Zugleich erlaubt die Anwendung des meaning-in-use Konzepts ein besseres und tiefergehendes Verständnis von Prozessen der Norminterpretation und Normanwendung.

Es zeigt sich also, dass der bereits angesprochene analytische Zugang zur Untersuchung von Wandel auch im konkreten Fall einen Erkenntnisgewinn mit sich bringt. Darüber hinaus ist dargelegt worden, dass die aus den theoretischen Überlegungen im ersten Abschnitt dieses Beitrags formulierte Annahme der Relevanz sozialer Praktiken sowie des Kontexts von Normen auch im konkreten Fall Bestand hat.

\section{Fazit}

Dieser Beitrag hat sich mit dem Verhältnis von Normativität und Normalität in der IB-Normenforschung auseinandergesetzt. Eine analytische Annäherung fand durch eine Perspektive des meaning-in-use statt. Die Prämisse dieses Ansatzes betont die Wichtigkeit sprachlicher und nicht-sprachlicher Interaktion für die Entstehung von Bedeutung von Normen. In Abgrenzung zu Forschungsansätzen, die vorrangig Fragen der Compliance untersuchen, kann hier nicht davon ausgegangen werden, dass Normalität ein

34 Saadi v Italy [2008] 24 B.H.R.C. 123, para 139.

35 Der Gerichtshof folgt hier also dem stare decidis-Grundsatz, was bedeutet, dass nachfolgende vorangegangenen Gerichtsentscheidungen nicht widersprechen dürfen. 
inhärentes Merkmal von Normen ist, welches beispielsweise auf Grundlage eines Verschriftlichungsprozesses letztgültig fixiert wurde.

Das Konzept des meaning-in-use betont vielmehr die Prozesshaftigkeit von Normen und ihrer Bedeutungen. Diese Eigenschaft von Normen, einem andauernden Entstehungs- und Entwicklungsprozess ausgesetzt zu sein, wurde anhand von zwei Fallbeispielen illustriert, die sich mit der Norm des Folterverbots auseinandergesetzt haben. Die Verschiebung von Normalität und Normativität des Folterverbots fand im Fall des US-Gefangenenlagers in Guantanamo Bay durch eine Re-Interpretation von Folter, im Fall der angesprochenen Fälle vor dem EGMR durch eine Re-Interpretation der Anwendungsbreite und Zielsetzung des Folterverbots in der Europäischen Menschenrechtskonvention statt. Zusammengenommen verweisen beide Fälle auf die Wirkungseffekte einer Neuinterpretation von Normen im Kontext der Bedrohung durch den internationalen Terrorismus. Während im Fall von Guantanamo durch eine zunächst gelungene Neuinterpretation eine neue Normalität geschaffen wird, zeigt die Fallgeschichte vor dem EGMR die entgegengesetzte Perspektive auf, also das Scheitern einer Neuauslegung.

Auf diese Weise ermöglicht meaning-in-use ein tiefergehendes Verständnis des Verhältnisses von Normativität und Normalität, welches sich weniger als spannungsgeladen denn als ein Miteinander darstellt. Daraus ergeben sich aus unserer Perspektive vier Konsequenzen.

Erstens rücken Praktiken und soziale Interaktion in den Mittelpunkt der Betrachtung eines kritischen Forschungsansatzes (Shapcott 2008: 334) jenseits von Compliance und formaler Validität ${ }^{36}$. Daher ermöglicht die hier erläuterte Perspektive Normenforschung jenseits positivistischer Rechtsvergleiche und dem Fokus auf Implementation und Compliance. In dieser Hinsicht steht der Beitrag dann auch stellvertretend für einen Pluralismus von Normenforschungen, wie ihn in diesem Sammelband u.a. Bastian Loges skizziert. Der Beitrag ist den monistischen, kontext-sensiblen Forschungsansätzen zuzurechnen (Jackson 2011).

Zweitens müssen Forschende methodologisch der Einsicht nachkommen, dass Praktiken stets ein »surplus of meaning «(Doty 1997: 377) besitzen, die sich sowohl dem menschlichen Streben nach Fixierung als auch einer angeblich stabilen (Sprach-)Struktur entziehen. Dies ist als doppelte Hermeneutik seit mindestens zwei Jahrzehnten auch in den IB bekannt (Guzzini 2000; Jackson 2006) und führt letztlich zu einem reflexiven Um-

36 Nicht ohne Grund gab es im vergangenen Jahrzehnt den sogenannten «practice turn»; siehe hierzu Neumann 2002, Pouliot 2008, Bueger/Gadinger 2008, 2018. 
gang mit Wissen: Anstatt zu Letztbegründungen führt die Untersuchung jeweils zu Zwischenerkenntnissen und erfährt keinen endgültigen Abschluss. Als Forschende, die für diese Thematik sensibilisiert sind, erheben wir zum einen nicht den Anspruch, einen Universalismus entdeckt zu haben. Zum anderen haben wir in den empirischen Beispielen so gut wie möglich versucht, Vordeutungen der Fälle zu unterlassen bzw. Heuristiken offen anzusprechen. Es wäre dann an Anderen oder an uns selbst, dies in einer weiteren Forschungsrunde zu hinterfragen. ${ }^{37}$

Was bei erster Betrachtung einen ungleich höheren Forschungsaufwand mit sich bringt, sehen wir aber, drittens, als eine Stärke des meaning-in-use Ansatzes. Er ermöglicht nämlich neue Fragestellungen und trägt dazu bei, vermeintlich gesichertes (Lehrbuch-)Wissen zu erschüttern. Es lässt sich nun nämlich die Frage stellen: Wie ist es möglich, dass die Verschiebung der Parameter des Folterverbots in liberalen Demokratien stattfindet (und dies kein Alleinstellungsmerkmal von Diktaturen, »Schurkenstaaten« und dergleichen ist)? Hiermit wird der Forschung eine Möglichkeit der Anknüpfung an Theoriedebatten eröffnet. Viertens wird für die Klärung dieser und ähnlich gelagerter Fragen zudem die Wichtigkeit inter- oder transdisziplinärer Forschung unterstrichen: Auch in den Rechtswissenschaften findet, analog zu den IB, häufig eine Analyse der formalen Validität statt, d.h. der kodifizierten Rechtsnorm, aber seltener eine Auseinandersetzung mit ihrer Entstehungsgeschichte und Interpretation (siehe aber Birkenkötter 2020 und Krisch/Corradini/Reimers 2020).

\section{Literatur}

Adler, Emanuel 2005: Communitarian International Relations: The Epistemic Foundations of International Relations, London.

Agamben, Giorgio 2005: State of Exception, Chicago, IL.

Abmad, Muneer I. 2009: Resisting Guantanamo: Rights at the Brink of Dehumanization, in: Northwestern University Law Review 103: 4, 1684-1764.

37 Eine Debatte hierzu fand vor einiger Zeit in der ZIB statt (Engelkamp et al. 2012, Ulbert 2012, Deitelhoff/Zimmermann 2013; Engelkamp et al. 2013; Hofius et al. 2014). Yanow (2009: 278-279) spricht sogar von der Notwendigkeit eines dritten hermeneutischen Moments zur Deutung und Beurteilung eines Forschungsgegenstands, um die Position der Forschenden reflexiv miteinzubeziehen (vgl. auch Hofius 2020: 176). 
Alkoby, Asher 2008: Theories of Compliance with International Law and the Challenge of Cultural Difference, in: Journal of International Law and International Relations 4: 1, 151-198.

Autesserre, Séverine 2014: Going Micro. Emerging and Future Peacekeeping Research, in: International Peacekeeping 21: 4, 492-500.

Berger, Peter/Luckmann, Thomas 1966: The Social Construction of Reality, London.

Birkenkötter, Hannah 2020: International Law as a Common Language Across Spheres of Authority?, in: Global Constitutionalism, 9: 2, 318-342.

Boerzel, Tanja A. 2001: Non-Compliance in the European Union: Pathology or Statistical Artefact?, in: Journal of European Public Policy 8: 5, 803-824.

Boerzel, Tanja A./Risse, Thomas 2002: Die Wirkung internationaler Institutionen. Von der Normanerkennung zur Normeinhaltung, in: Jachtenfuchs, Markus/ Knodt, Michèle (Hrsg.): Regieren in internationalen Institutionen, Wiesbaden, 141-181.

Brunnée, Jutta/Toope, Stephen J. 2010: Legitimacy and Legality in International Law, Cambridge.

Bueger, Christian 2014: Pathways to Practice: Praxiography and International Politics, in: European Political Science Review 6: 3, 383-406.

Bueger, Christian/Gadinger, Frank 2008: Praktisch Gedacht! Praxistheoretischer Konstruktivismus in den Internationalen Beziehungen, in: Zeitschrift für Internationale Beziehungen 15: 2, 273-302.

Bueger, Christian/Gadinger, Frank 2018: International Practice Theory, 2nd edition, Houndmills, Basingstoke, Hampshire.

Burchill, Scott/Devetak, Richard/Linklater, Andrew/Paterson, Matthew/Reus-Smit, Christian/True, Jacqui (Hrsg.) 2003: Theories of International Relations, 2. Auflage, Basingstoke.

Butler, Judith 1997: Excitable Speech: A Politics of the Performative, New York, NY.

Bybee, Jay S. 2002: Memorandum from Jay S. Bybee, Assistant Attorney Gen., Office of Legal Counsel, U.S. Department of Justice, to an Alberto R. Gonzales, Counsel to the President, Re: Standards of Conduct for Interrogation Under 18 U.S.C. $\$ \$$ 2340-2340A (Aug. 1, 2002), in: http://www.nytimes.com/ref/international/24MEMO-GUIDE.html?_r=0; 19.1.2021.

Chayes, Abram/Chayes, Antonia 1995: The New Sovereignty: Compliance with International Regulatory Agreements, Cambridge, MA.

Coulter, Jeff 2001: Human Practices and the Observability of the 'Macro-Social', in: Schatzki, Theodore R./Knorr-Cetina, Karin/von Savigny, Eike (Hrsg.): The Practice Turn in Contemporary Theory, New York, NY, 29-41.

Cover, Robert 1983: The Supreme Court 1982, Foreword: Nomos and Narrative, in: Harvard Law Review 97: 4, 4-68.

Cox, Robert W. 2008: The Point is not Just to Explain the World but to Change it, in: Reus-Smit, Christian/Snidal, Duncan (Hrsg.): The Oxford Handbook of International Relations, Oxford, 84-93. 
Deitelhoff, Nicole/Zimmermann, Lisbeth 2013: Aus dem Herzen der Finsternis: Kritisches Lesen und wirkliches Zuhören der konstruktivistischen Normenforschung, in: Zeitschrift für Internationale Beziehungen 20: 1, 61-74.

Deitelhoff, Nicolel Zimmermann, Lisbeth 2020: "Things We Lost in the Fire: How Different Types of Contestation Affect the Robustness of International Norms.” International Studies Review 22: 1, 51-76.

Doty, Roxanne L. 1997: Aporia: A Critical Exploration of the Agent-Structure Problematique in International Relations Theory, in: European Journal of International Relations 3: 3, 365-392.

Dunne, Tim/Kurki, Milja/Smith, Steve (Hrsg.) 2010: International Relations Theory: Discipline and Diversity, 2. Auflage, Oxford.

Engelkamp, Stephan/Glaab, Katharina/Renner, Judith 2012: In der Sprechstunde. Wie (kritische) Normenforschung ihre Stimme wiederfinden kann, in: Zeitschrift für Internationale Beziehungen 19: 2, 101-128.

Engelkamp, Stephan/Glaab, Katharina/Renner, Judith 2013: Ein Schritt vor, zwei Schritte zurück? Eine Replik auf Nicole Deitelhoff und Lisbeth Zimmermann, in: Zeitschrift für Internationale Beziehungen 20: 2, 105-118.

EMRK 1950: Europäische Menschenrechtskonvention vom 4. November 1950, Rom.

Fabian, Johannes 1990: Power and Performance. Ethnographic Explorations through Proverbial Wisdon and Theater in Shaba, Zaire, Madison, WI.

Fierke, Karin M. 2001: Critical Methodology and Constructivism, in: Fierke, Karin/ Jørgensen, Knud E. (Hrsg.): Constructing International Relations: The Next Generation, Armonk, NY, 115-135.

Fierke, Karin M. 2010: Constructivism, in: Dunne, Tim/Kurki, Milja/Smith, Steve (Hrsg.): International Relations Theory: Discipline and Diversity, Oxford, 177-194.

Fierke, Karin M./Jørgensen, Knud E. (Hrsg.) 2001: Constructing International Relations: The Next Generation, Armonk, NY.

Fierke, Karin M./Wiener, Antje 1999: Constructing Institutional Interests: EU and NATO Enlargement, in: Journal of European Public Policy 6: 5, 721-742.

Finnemore, Martha 2000: Are Legal Norms Distinctive?, in: Journal of International Law \& Politics 32: 3, 699-705.

Finnemore, Martha/Sikkink, Kathryn 1998: International Norm Dynamics and Political Change, in: International Organization 52: 4, 887-917.

Foucault, Michel 1977: Discipline and Punish: The Birth of the Prison, New York, NY.

Garfinkel, Harold 1967: Studies in Ethnomethodology, Cambridge.

Geblen, Dirk von 2012: Die normative Kraft des Faktischen, in: Süddeutsche Zeitung, 6.2.2012, 11.

Gregory, Derek 2006: The Black Flag: Guantánamo Bay and the Space of Exception, in: Geografiska Annaler: Series B, Human Geography 88: 4, 405-427. 
Guzzini, Stefano 2000: A Reconstruction of Constructivism in International Relations, in: European Journal of International Relations 6: 2, 147-182.

Hansen-Magnusson, Hannes 2020: International Relations as Politics among People. Hermeneutic Encounters and Global Governance, Abingdon and New York.

Hofius, Maren 2016: Community at the Border or the Boundaries of Community? The Case of EU Field Diplomats, in: Review of International Studies 42: 5, 939967.

Hofius, Maren 2020: Towards a 'Theory of the Gap': Addressing the Relationship Between Practice and Theory, in: Global Constitutionalism 9: 1, 169-182.

Hofius, Maren/Wilkens, Jan/Hansen-Magnusson, Hannes/Gholiagha, Sassan 2014: Den Schleier lichten? Kritische Normenforschung, Freiheit und Gleichberechtigung im Kontext des "Arabischen Frühlings", in: Zeitschrift für Internationale Beziehungen 21: 2, 85-105.

Holzscheiter, Anna 2014: Between Communicative Interaction and Structures of Signification: Discourse Theory and Analysis in International Relations, in: International Studies Perspective 15: 2, 142-162.

Holzscheiter, Anna 2017: Was vom arguing übrigblieb...: Der Nachhall der kommunikativen Wende in den Internationalen Beziehungen, in Zeitschrift für Internationale Beziehungen 24: 1, 143-159.

Hopf, Ted 1998: The Promise of Constructivism in International Relations Theory, in: International Security 23: 1, 171-200.

Howse, Robert/Teitel, Ruti 2010: Beyond Compliance: Rethinking Why International Law Really Matters, in: Global Policy 1: 2, 127-136.

Hunt, Charles T./Orchard, Phil (Hrsg.) 2020: Constructing the Responsibility to Protect: Contestation and Consolidation. Abingdon, Oxon.

Jachtenfuchs, Markus/Knodt, Michèle (Hrsg.) 2002: Regieren in internationalen Institutionen, Wiesbaden.

Jackson, Patrick Thaddeus (2006): Making Sense of Making Sense: Configurational Analysis and the Double Hermeneutic. Interpretation and Method: Empirical Research Methods and the Interpretive Turn. D. Yanow and P. Schwartz-Shea. Armonk, NY: 264-280.

Jackson, Patrick Thaddeus (2011): The Conduct of Inquiry in International Relations: Philosophy of Science and Its Implications for the Study of World Politics. London; New York.

Janis, Mark W./Kay, Richard S./Bradley, Anthony W. 2008: European Human Rights Text and Materials, 3. Auflage, Oxford.

Jepperson, Ronald L./Wendt, Alexander/Katzenstein, Peter J. 1996: Norms, Identity, and Culture in National Security, in: Katzenstein, Peter J. (Hrsg.): The Culture of National Security: Norms and Identity in World Politics, New York, 33-75.

Jileva, Elena 2004: Do Norms Matter? The Principle of Solidarity and the EU's Eastern Enlargement, in: Journal of International Relations and Development 7: 1, $3-23$. 
Joerges, Christian 2005: Confronting Memories: European "Bitter Experiences" and the Constitutionalization Process. Constructing Europe in the Shadow of its Pasts, in: German Law Journal 6: 2, 245-254.

Johns, Fleur 2005: Guantánamo Bay and the Annihilation of the Exception, in: European Journal of International Law 16: 4, 613-635.

Katzenstein, Peter J. 1996: Introduction: Alternative Perspectives on National Security, in: Katzenstein, Peter J. (Hrsg.): The Culture of National Security: Norms and Identity in World Politics, New York, NY, 1-32.

Katzenstein, Peter J. (Hrsg.) 1996: The Culture of National Security: Norms and Identity in World Politics, New York, NY.

Klabbers, Jan 2006: The Meaning of Rules, in: International Relations 20: 3, 295-301.

Kratochwil, Friedrich 1989: Rules, Norms, and Decisions: On the Conditions of Practical and Legal Reasoning in International Relations and Domestic Affairs, Cambridge.

Kratochwil, Friedrich/Ruggie, John G. 1986: International Organization: A State of the Art on an Art of the State, in: International Organization 40: 4, 753-775.

Krisch, Nico/Corradini, Francesco/Reimers, Luci Lui. 2020: Order at the Margins: The Legal Construction of Interface Conflicts over Time, in Global Constitutionalism, 9: 2: 343-363.

Laffey, Mark/Weldes, Jutta 1997: Beyond Belief: Ideas and Symbolic Technologies in International Relations, in: European Journal of International Relations 3: 2, 193-237.

Lantis, Jeffrey S./Wunderlich, Carmen 2018: Resiliency Dynamics of Norm Clusters: Norm Contestation and International Cooperation, in Review of International Studies 44: 3, 570-593.

Legro, Jeffrey W. 1997: Which Norms Matter? Revisiting the 'Failure' of Internationalism, in: International Organization 51: 1, 31-63.

Liese, Andrea 2009: Exceptional Necessity: How Liberal Democracies Contest the Prohibition of Torture and Ill-Treatment when Countering Terrorism, in: Journal of International Law and International Relations 5: 1, 17-48.

Milliken, Jennifer 1999: The Study of Discourse in International Relations: A Critique of Research and Methods, in: European Journal of International Relations 5: 2, 225-254.

Moeckli, Daniel 2005: Saadi v. Italy: The Rules of the Game Have Not Changed, in: Human Rights Law Review 8: 3, 534-548.

Neal, Andrew W. 2006: Foucault in Guantánamo: Towards an Archaeology of the Exception, in: Security Dialogue 37: 1, 31-46.

Neufeld, Mark 1993a: Interpretation and the 'Science' of International Relations, in: Review of International Studies 19: 1, 39-61.

Neufeld, Mark 1993b: Reflexivity and International Relations Theory, in: Millennium: Journal of International Studies 22: 1, 53-76. 
Neumann, Iver B. 2002: Returning Practice to the Linguistic Turn: The Case of Diplomacy, in: Millennium - Journal of International Studies, 31: 3, 627-651.

Niemann, Holger/Schillinger, Henrik 2017: Contestation 'All the Way Down'? The Grammar of Contestation in Norm Research, in: Review of International Studies 43: 1: 29-49.

Nollkaemper, André 2012: The ECtHR Finds Macedonia Responsible in Connection with Torture by the CIA, But on What Basis?, in: http://www.ejiltalk.org/the-ecthr-finds-macedonia-responsible-in-connection-with-torture-by-the-cia-but-onwhat-basis; 19.1.2021.

Nowak, Manfred 2005: Challenges to the Absolute Nature of the Prohibition of Torture and Ill-Treatment, in: Netherlands Quarterly of Human Rights 23: 4, 674-688.

Onuf, Nicholas G. 1989: World of Our Making. Rules and Rule in Social Theory and International Relations, New York, NY.

Palmer, Stephanie 2006: A Wrong Turning: Article 3 ECHR and Proportionality, in: Cambridge Law Journal 65: 2, 438-452.

Park, Susan/Vetterlein, Antje (Hrsg.) 2010: Owning Development. Creating Global Policy Norms in the IMF and the World Bank, Cambridge.

Porth, Jacquelyn S. 2004: Pentagon Creates New Policy Office to Review Detainee Issues, in: http://www.iwar.org.uk/news-archive/2004/07-16.htm; 20.1.2021.

Pouliot, Vincent 2008: The Logic of Practicality: A Theory of Practice of Security Communities, in: International Organization 62: 2, 257-288.

Puetter, Uwe/Wiener, Antje 2009: Quality of Norms is What Actors Make of It: Critical-Constructivist Research on Norms, in: Journal of International Law and International Relations 5: 1, 1-16.

Reus-Smit, Christian 2003: Constructivism, in: Burchill, Scott/Devetak, Richard/ Linklater, Andrew/Paterson, Matthew/Reus-Smit, Christian/True, Jacqui (Hrsg.): Theories of International Relations, Basingstoke, 188-212.

Reus-Smit, Christian/Snidal, Duncan (Hrsg.) 2008: The Oxford Handbook of International Relations, Oxford.

Risse, Thomas/Ropp, Stephen C./Sikkink, Kathryn 1999: The Power of Human Rights: International Norms and Domestic Change, Cambridge.

Ruby, Jay (Hrsg.) 1982: A Crack in the Mirror: Reflexive Perspectives in Anthropology, Philadelphia, PA.

Sandholtz, Wayne 2019: Norm Contestation, Robustness, and Replacement, in Journal of Global Security Studies, 4: 1, 139-146

Schatzki, Theodore R./Knorr-Cetina, Karin/von Savigny, Eike (Hrsg.) 2001: The Practice Turn in Contemporary Theory, New York, NY.

Shapcott, Richard 2008: Critical Theory, in: Reus-Smit, Christian/Snidal, Duncan (Hrsg.): The Oxford Handbook of International Relations, Oxford, 327-345.

Smith, Steve/Booth, Ken/Zalewski, Marysia (Hrsg.) 1996: International Theory: Positivism \& Beyond, Cambridge. 
Solomon, Ty, Brent J. Steele 2017: Micro-Moves in International Relations Theory, in: European Journal of International Relations 23: 2, 267-291.

Spiegel Online 2004: Ehrenwerte Motive, mildes Urteil, in: http://www.spiegel.de/pa norama/daschner-prozess-ehrenwerte-motive-mildes-urteil-a-333706.html; 19.1.2021.

Steyn, Johan 2004: Guantanamo Bay: The Legal Black Hole, in: International \& Comparative Law Quarterly 53: 1, 1-15.

Stone Sweet, Alec 2012: A Cosmopolitican Legal Order: Constitutional Pluralism and Rights Adjucation in Europe, in: Global Constitutionalism - Human Rights, Democracy, and the Rule of Law 1: 1, 53-90.

Turner, Victor 1982: Dramatic Ritual/Ritual Drama: Performative and Reflexive Anthropology, in: Ruby, Jay (Hrsg.): A Crack in the Mirror. Reflexive Perspectives in Anthropology, Philadelphia, PA, 83-97.

Ulbert, Cornelia 2012: Vom Klang vieler Stimmen: Herausforderungen »kritischer« Normenforschung. Eine Replik auf Stephan Engelkamp, Katharina Glaab und Judith Renner, in: Zeitschrift für Internationale Beziehungen 19: 2, 129-130.

US Department of Defence 2004: Memorandum for the Secretary of the Navy: Order Establishing Combatant Status Review Tribunal, Washington DC.

Venzke, Ingo 2009: Legal Contestation about 'Enemy Combatants': On the Exercise of Power in Legal Interpretation, in: Journal of International Law and International Relations 5: 1, 157-184.

Waver, Ole 1996: The Rise and Fall of the Inter-Paradigm Debate, in: Smith, Steve/ Booth, Ken/Zalewski, Marysia (Hrsg.): International Theory: Positivism \& Beyond, Cambridge, 149-185.

Welsh, Jennifer 2013: Norm Contestation and the Responsibility to Protect, in: Global Responsibility to Protect 5, 365-396.

Weldes, Jutta 1998: Bureaucratic Politics: A Critical Constructivist Assessment, in: Mershon International Studies Review 42: 2, 216-225.

Weldes, Jutta/Saco, Diana 1996: Making State Action Possible: The United States and the Discursive Construction of 'The Cuban Problem', 1960-1994, in: Millennium: Journal of International Studies 25: 2, 361-395.

Wendt, Alexander 1992: Anarchy Is What States Make of It: The Social Construction of Power Politics, in: International Organization 46: 2, 391-426.

Wendt, Alexander 1998: On Constitution and Causation in International Relations, in: Review of International Studies 24: 5, 101-117.

Wiener, Antje 2004: Contested Compliance: Interventions on the Normative Structure of World Politics, in: European Journal of International Relations 10: 2, 189-234.

Wiener, Antje 2008: The Invisible Constitution of Politics: Contested Norms and International Encounters, Cambridge.

Wiener, Antje 2009: Enacting Meaning-in-Use: Qualitative Research on Norms and International Relations, in: Review of International Studies 35: 1, 175-193. 
Wiener, Antje 2010: Normative Baggage in International Encounters: Contestation all the Way., in: Kessler, Oliver/Hall, Rodney Bruce/ Lynch, Cecilia/Onuf, Nicholas (Hrsg.) On Rules, Politics, and Knowledge: Friedrich Kratochwil, International Relations, and Domestic Affairs, Basingstoke, New York, Palgrave Macmillan, 202-212.

Wiener, Antje 2014: A Theory of Contestation, Berlin.

Wiener, Antje 2018: Contestation and Constitution of Norms in Global International Relations, Cambridge.

WVRK 1969: Wiener Vertragsrechtskonvention vom 23. Mai 1969, Wien.

Yanow, Dvora 2009: Dear Author, Dear Reader: The Third Hermeneutic in Writing and Reviewing Ethnography, in: Schatz, Edward (Hrsg.): Political Ethnography. What Immersion Contributes to the Study of Power, Chicago, London 2009, 275-302. 
